\title{
Normal Displacements on a Reconstructed Silicon (111) Surface: An X-Ray-Standing-Wave Study
}

\section{Citation}

Patel, J. R., P. E. Freeland, J. A. Golovchenko, A. R. Kortan, D. J. Chadi, and Guo -Xin Qian. 1986. Normal Displacements on a Reconstructed Silicon (111) Surface: An X-Ray-Standing-Wave Study. Physical Review Letters 57, no. 24: 3077-3080. doi:10.1103/physrevlett.57.3077.

\section{Published Version}

doi:10.1103/PhysRevLett.57.3077

\section{Permanent link}

http://nrs.harvard.edu/urn-3:HUL.InstRepos:29407042

\section{Terms of Use}

This article was downloaded from Harvard University's DASH repository, and is made available under the terms and conditions applicable to Other Posted Material, as set forth at http:// nrs.harvard.edu/urn-3:HUL.InstRepos:dash.current.terms-of-use\#LAA

\section{Share Your Story}

The Harvard community has made this article openly available.

Please share how this access benefits you. Submit a story.

\section{Accessibility}




\title{
Normal Displacements on a Reconstructed Silicon (111) Surface: An X-Ray-Standing-Wave Study
}

\author{
J. R. Patel, P. E. Freeland, J. A. Golovchenko, and A. R. Kortan \\ AT\& $T$ Bell Laboratories, Murray Hill, New Jersey 07974 \\ and \\ D. J. Chadi and Guo-Xin Qian \\ Xerox Palo Alto Research Center, Palo Alto, California 94034 \\ (Received 11 June 1986)
}

\begin{abstract}
The average relaxation of the top layers of a germanium-atom-tagged $\mathrm{Si}(111)$ surface has been measured by $x$-ray interferometric and fluorescence methods. The results indicate a slight outward displacement $(\sim 2 \%)$ of the outer layers on a $(5 \times 5)$ reconstructed surface relative to a perfectly terminated silicon crystal surface. The results are in good quantitative agreement with atom positions predicted from a tight-binding-based energy-minimization calculation of the dimer-adatom stacking-fault model of the $\mathrm{Si}(111)(7 \times 7)$ or $(5 \times 5)$ surface.
\end{abstract}

PACS numbers: $68.35 . \mathrm{Bs}, 61.10 . \mathrm{Lx}, 61.70 . \mathrm{Ph}, 68.35 . \mathrm{Dv}$

A major change in attitude concerning the nature of the equilibrium $\mathrm{Si}(111)$ surface has occurred over the past few years within the surface physics community. Studies using the powerful methods of transmission electron diffraction, ${ }^{1,2} \mathrm{x}$-ray diffraction, ${ }^{3}$ and tunneling microscopy ${ }^{4,5}$ have yielded results that are highly consistent with the "dimer, adatom, stacking fault (DAS)" model of Takayanagi.' From a diffraction point of view, the existence of three independent diffraction determinations helps to make a convincing argument that the DAS model explains the distribution of matter parallel to the surface in the $(7 \times 7)$ reconstruction. Reliable structural information on the distribution of surface atoms normal to the crystal now is essential to unite theory and experiment in a complete solution to this long-standing and important surface-physics problem. Using a surface decoration technique and $\mathrm{x}$-ray standing waves in an ultrahigh-vacuum (UHV) environment, we have arrived at a measure of the average distribution of surface atoms normal to the (111) reconstructed surface. Enough confidence in the DAS model has been generated to stimulate increasingly complex and sophisticated calculations of the electronic structure and total energy of the surface. ${ }^{6}$ Results of these calculations show significant relaxations of the adatoms and the atoms directly below them. We will demonstrate for the first time that there is good quantitative agreement between theoretically predicted atom positions normal to the surface and those determined with $\mathrm{x}$-ray standing waves.

In previous studies we have demonstrated that impurity coverages in the monolayer range can be studied accurately with $x$-ray standing waves. ${ }^{7-9}$ The standing-wave technique first pioneered by Batterman ${ }^{10}$ was later developed into a highly accurate interferometric technique of locating both bulk ${ }^{10-12}$ and surface atom positions. ${ }^{7-9}$ The standard inelastic signal used in most pre- vious $\mathrm{x}$-ray standing-wave studies has been derived from $x$-ray fluorescence. The large depth from which fluorescent $x$ rays emanate would normally preclude their use as a probe for measuring surface reconstructions of atoms of the same species as those which constitute the bulk. We have circumvented this problem by replacing the top layer of silicon atoms on the surface with germanium atoms. The detection of secondary Ge $K \alpha \mathrm{x}$ rays excited by the $x$-ray standing wave then corresponds to a surface-specific probe. It may legitimately be argued that replacing the topmost silicon layers with a foreign atom even of the same chemical group may significantly alter the silicon $(111)(7 \times 7)$ reconstruction. We argue here that any change in the arrangement of atoms due to a top layer of germanium is very minor perturbation of the $\mathrm{Si}(7 \times 7)$, for the purposes of this study. To start with, the $(7 \times 7)$ reconstruction on a silicon (111) surface is not unique; it belongs to the general class of reconstructions $(5 \times 5),(7 \times 7)$, and $(9 \times 9)$, all of which have been observed on a silicon (111) surface. ${ }^{13,14}$ Important for our present studies is the observation that the $(7 \times 7)$ reconstruction persists when monolayer (ML) coverages of $\mathrm{Ge}$ are rapidly $(1 \mathrm{ML} / \mathrm{sec})$ deposited on a silicon (111) surface. $^{15}$ Indeed, though it is well known that clean surfaces of bulk germanium show a $(2 \times 8)$ reconstruction, a high rate of deposition of germanium on a $\mathrm{Si}(111)(7 \times 7)$ surface causes it to adopt the $(7 \times 7)$ reconstruction up to film thicknesses of 500-1000 $\AA .^{15}$ At slower rates of deposition from ordinary effusion cells (1 ML/300 sec) the closely related $(5 \times 5)$ reconstruction is observed in this study and other investigations. ${ }^{16}$ An added argument that perturbation due to $\mathrm{Ge}$ on $\mathrm{Si}(7 \times 7)$ is slight is the observation that elements not in the same chemical group drastically alter the $(7 \times 7)$ reconstruction at submonolayer coverages. ${ }^{17,18}$

The details of the $x$-ray part of our experiment have 


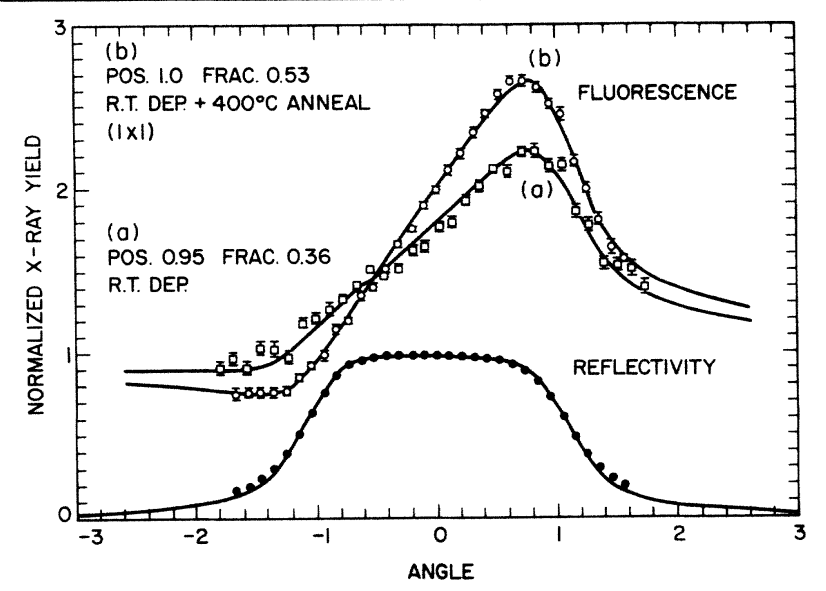

FIG. 1. Reflectivity and fluorescence yield for $\mathrm{Ge}$ on $\mathrm{Si}(111)$ surface (curve $a$ ) after room temperature deposition, (curve $b$ ) after $400^{\circ} \mathrm{C}$ anneal. Solid curves are fits by dynamical theory.

been reported elsewhere for a very similar nonvacuum study of the silicon-amorphous-silicon interface. ${ }^{7}$ The main difference here is that the tedious problems of performing the experiment in a UHV environment with in situ surface preparation have been solved. Although further work remains to be done we believe our results will be of interest to theorists and experimentalists alike.

The silicon sample was cleaned with a Shiraki etch ${ }^{19}$ prior to mounting in the UHV chamber. This etch has been shown to result in a minimum amount of carbon contamination of the sample surface which then requires only a low-temperature heating $\left(800-900^{\circ} \mathrm{C}\right)$ to form a $(7 \times 7)$ reconstructed surface under UHV $\left(10^{-10}\right.$ torr $)$ conditions. After a $(7 \times 7)$ reconstruction was confirmed by LEED (low-energy electron diffraction) the sample was placed opposite a precleaned and out-gassed germanium effusion cell. A monolayer $\left(\sim 8 \times 10^{14}\right.$ atoms/ $\mathrm{cm}^{2}$ ) of germanium was deposited on the sample over a period of $5 \mathrm{~min}$. As in earlier work $^{7}$ on amorphouscapped crystals, we will demonstrate that the germanium atoms occupy both sublattice atom positions available on (111) planes at the surface. Hence, 1-ML coverage would result in an effective coverage of $\frac{1}{2}$ of the silicon surface. The coverage was determined with an Auger spectrometer calibrated for germanium $L M M$ sensitivity by Rutherford backscattering. The silicon $L M M$ line was also monitored after deposition to verify that neither islanding nor indiffusion accompanied the surface doping. ${ }^{16}$

Following a room-temperature deposition of a monolayer of germanium the surface was no longer observed to produce any LEED spots at all. The first $x$-ray standing-wave result reported here was taken under this condition (Fig. 1, curve a). After a $400^{\circ} \mathrm{C}$ anneal a $(1 \times 1)$ pattern was observed with LEED and a second $\mathrm{x}$-ray measurement (always at room temperature) was

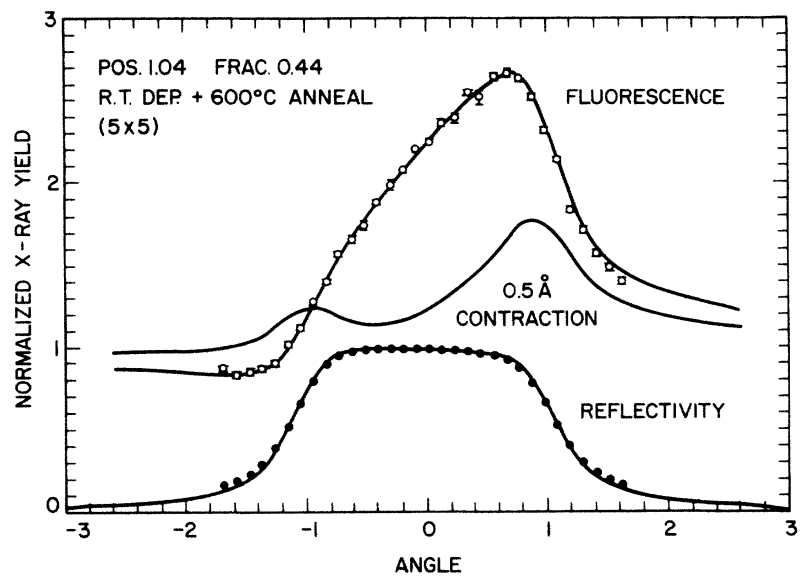

FIG. 2. Reflectivity and fluorescence yield for $\mathrm{Ge}$ on $\mathrm{Si}(111)$ surface after $600^{\circ} \mathrm{C}$ anneal. Solid curves are fits by dynamical theory.

taken (Fig. 1, curve $b$ ). A subsequent anneal to $600^{\circ} \mathrm{C}$ resulted in a $(5 \times 5)$ pattern on the LEED apparatus. This was the highest temperature that would not result in significant diffusion of germanium into the sample or evaporation from it. A third $\mathrm{x}$-ray measurement was taken for this preparation (Fig. 2). A final $\mathrm{x}$-ray result was obtained under ambient conditions for germanium completely diffused in from the surface layer, yet near enough to the surface $(<1000 \AA)$ to eliminate all $x$-ray extinction corrections when we interpreted the data (Fig. 3 ). The sequential $x$-ray fluorescence and Bragg reflectivity results for each of the above experiments are presented in Figs. 1-3. For good statistics the duration of a typical $\mathrm{x}$-ray standing-wave run was $16 \mathrm{~h}$. However, results obtained with poorer statistics after $6 \mathrm{~h}$ did not show any significant change after the longer-duration

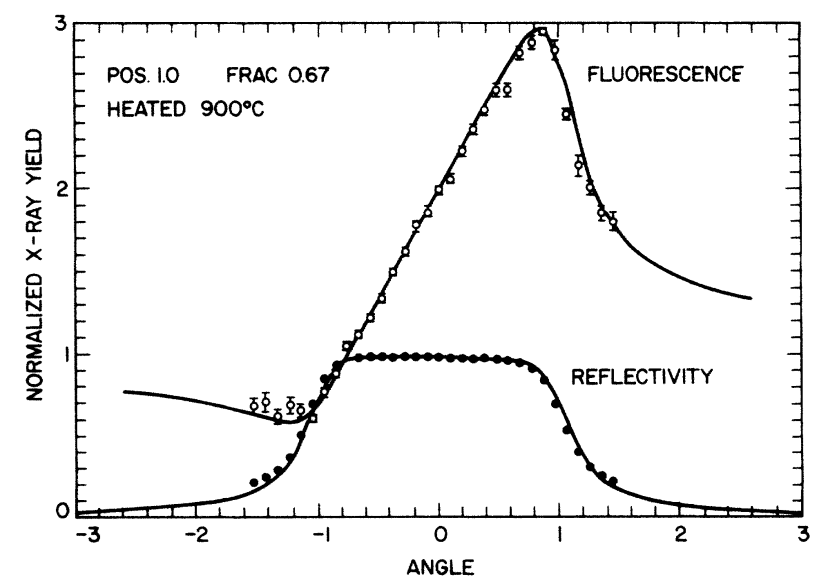

FIG. 3. Reflectivity and fluorescence yield for Ge diffused into $\mathrm{Si}$ at $900^{\circ} \mathrm{C}$ for $0.5 \mathrm{~h}$. Solid curves are fits by dynamical theory. 
measurements. Neither the Auger-electron spectroscopy (AES) or LEED pattern showed any observable change after the standing-wave measurements.

A powerful feature of the standing-wave method, as pointed out in Ref. 8 , is that it provides a direct measure of both amplitude and phase of the atomic-density Fourier component being sampled, which is the (111) component in this case. As has been customary in lattice location studies we reference the phase of the impurity or bulk atom-density Fourier component relative to that of the same Fourier component of the bulk electron density. The latter has a maximum between the closely spaced (111) atomic planes and this position serves as our phase reference. The phase variable for the germanium surface density is labeled "POS" in the figures and this is the phase of the impurity distribution converted into a normalized position relative to the aforementioned reference point. The normalization is such that $\operatorname{POS}=1.0$ corresponds to the first unrelaxed extrapolation of the last silicon surface plane by one (111) period spacing (3.14 Å). Smaller values of POS correspond to an inwardly relaxed Fourier component and larger ones to an outward relaxation. The amplitude of the Fourier component is contained in the variable labeled FRAC in the figures which stands for coherent fraction. It denotes the fraction of the atoms that would have to be at position POS to yield a signal of the strength observed. We now discuss the experimental results in these terms. It will be seen from the figures that the quality of the fluorescence and reflection curves is quite good, in terms of both the angular resolution and statistics. The fits to theory with use of POS and FRAC as adjustable parameters are also quite good with errors in these essentially orthogonal parameters of order $1 \%$.

Right after deposition at room temperature (no anneal) the germanium POS value is 0.95 corresponding to a $5 \%$ or $0.15-\AA$ surface contraction (Fig. 1, curve $a$ ). After the $400^{\circ} \mathrm{C}$ anneal the contraction disappears with POS $=1.0$ (Fig. 1, curve $b$ ). After annealing to $600^{\circ} \mathrm{C}$ where the $(5 \times 5)$ reconstructions appears there is a $4 \%$ expansion of the surface layer since POS is $1.04 \pm 0.01$. Shown in Fig. 2 for comparison purposes is the curve expected if the top layers of the reconstructed surface were contracted by $0.5 \AA$ (POS $=0.82)$. Such a value has recently been reported by Durbin et al. ${ }^{20}$ for reconstructed untagged silicon and will be discussed below. Figure 3 shows that the diffused sample has values of POS and FRAC of 1.0 and 0.67 , respectively, in good agreement with the values of 1.0 and 0.7 expected of bulk substitutional germanium.

The experimental finding can now be tested against current models of the $(7 \times 7)$ and closely related $(5 \times 5)$ reconstruction. What we need is a theoretical prediction of the Fourier components of the normal distribution of the surface atoms. Toward this end we have used the results of a semiempirical tight-binding-based energy- minimization calculation. To accommodate the substantial energy shifts resulting from the adatom and the atoms directly below, results of a self-consistent pseudopotential density-functional calculation were incorporated in the total energy calculations. For the DAS model of the silicon $(111)(7 \times 7)$ surface a four-layer slab plus the adatom layer was considered. There was a total of 249 atoms included in the calculation. HellmannFeynman forces were calculated in each step of the interaction process to determine the direction of motion of atoms which would result in a lowering of the total energy. In this way results were obtained on the final geometry of the completely relaxed DAS model for the first three layers and for the adatom layer. The surface energy of the completely relaxed silicon (111) $(7 \times 7)$ structure was also calculated and was found to be only $2 \%$ lower than a similar calculation for the silicon (111) $(5 \times 5)$ reconstruction. The overall results, whose accuracy may be trusted to $0.1 \AA$, are in close agreement with those expected from the DAS model as regards the transverse atomic coordinates. A Fourier analysis of the top double layer and adatom normal coordinates yields theoretical values of POS $=0.96$ and FRAC $=0.42$ for both $(7 \times 7)$ and $(5 \times 5)$ structures. This corresponds to a $4 \%$ or $0.12-\AA$ contraction. As far as the displacements normal to (111) are concerned, a Fourier analysis of the calculations yields identical POS and FRAC results for both $(7 \times 7)$ and $(5 \times 5)$ reconstructions. This reinforces our earlier arguments that comparisons of the normal displacements of $(7 \times 7)$ with $(5 \times 5)$ reconstructions are valid.

Comparison with theory is slightly complicated by the presence of germanium rather than silicon atoms near the surface used in the calculation. Germaniumgermanium covalent bonds lengths are $4 \%$ larger than those for silicon-silicon bonds. Germanium-silicon bonds are perhaps $2 \%$ larger. We may therefore expect our experiments to yield a $2 \%-4 \%$ expansion over that expected for a silicon-terminated sample. When we take this and the uncertainties associated with both experiment and theory into account, the overall agreement supports the DAS model quite well.

The recent experimental report by Durbin et al. ${ }^{20}$ that the top double layer of a $\mathrm{Si}(111)(7 \times 7)$ surface has a mean normal surface contraction of $0.5 \AA$ is quite significant in the context of the present report. To limit the signal to a small depth near the surface these authors have used the inelastic Auger channel. A number of points concerning the difference between our experiment and that of Durbin et $a .^{20}$ must now be addressed because they might account for the $0.5-\AA$ discrepancy between the results. First, the germanium atoms participating in the reconstruction of our surface are in the $(5 \times 5)$ configuration and thus our results may not be directly comparable with those for a $(7 \times 7)$ surface. Both $(7 \times 7)$ and $(5 \times 5)$ surfaces have, however, recently 
been studied by tunneling microscopy ${ }^{21}$ and transmission electron diffraction ${ }^{22}$ and appear to be essentially identical with regard to the transverse distribution of matter except for a lateral shrinkage of the unit cell obtained, not by a change in surface atom positions, but by a decrease in the number contained in the unit cell. The transverse structure of both reconstructions can be straightforwardly accounted for within the DAS model. ${ }^{22}$ Recent tunneling microscope measurements we have performed $^{14}$ on laser-annealed and partially thermalannealed surfaces containing both $(5 \times 5)$ and $(7 \times 7)$ reconstructions on silicon (111) surfaces show no height differences between the reconstructions in agreement with the results of the calculations for the DAS models described above, which give the same POS and FRAC values for $(5 \times 5)$ and $(7 \times 7)$. Second, the differences between the germanium and silicon top layers in the two experiments might explain the discrepancy, but other than shifting the reconstruction to the nearby $(5 \times 5)$, bond-length differences of only a few percent would be anticipated, as previously discussed.

So why do the results of Durbin et al. disagree with those presented here? We suggest that there may be several reasons. First, the possibility of incoherent signals, resulting from unreconstructed and disordered regions of the sample, was not considered in the analysis of those data. Such signals would constitute different fractions of the two Auger signals compared in that work because of the different escape depths involved, and on the basis of the analysis shown, mimic a surface layer contraction. If $f_{2}$ values $<1$ had been chosen in Eq. (1) of Ref. 18, different values of contraction would have been deduced. Another contribution to uncertainty comes from the background subtraction necessary to eliminate interfering effects of $K L L$ Auger electrons originating deep in the sample from the $L M M$ Auger peak. There are two possible contributions here, one being from $L M M$ 's excited by $K L L$ 's and the other simply from $K L L$ 's whose energy is degraded during escape from the sample. The depth in the sample at which the initial $K L L$ electron is born will in both cases result in extinction effects which also incorrectly mimic a lattice contraction. This is because when extinction is present, the simple theory of standing-wave effects at the surface is no longer applicable. A more detailed theory to take into account signals from bulk atoms such as that used by Batterman ${ }^{10}$ invariably suppresses the yield within the total-reflection region in a way that would be interpreted as a contraction in the simple theory.

We conclude that for the reconstructed surface studied here by $x$-ray standing-wave interferometric methods there is at most a slight outward shift of the surfacelayer (111) Fourier component. When we take into ac- count simple atomic size effects associated with substitution of germanium for silicon atoms, this result is in satisfactory agreement with and supports the DAS model for the reconstruction of $\mathrm{Si}(111)$ surfaces.

We are grateful to E. G. McRae and L. C. Kimerling for critical comments.

${ }^{1}$ K. Takayanagi, Y. Tanishiro, M. Takahashi, and S. Takahashi, J. Vac. Sci. Technol. A 3, 1502 (1985).

${ }^{2}$ E. G. McRae and P. M. Petroff, Surf. Sci. 147, 385 (1984).

${ }^{3}$ I. K. Robinson, W. K. Waskiewicz, P. H. Fuoss, J. B. Stark, and P. A. Bennett, Phys. Rev. B 33, 7013 (1986).

${ }^{4}$ J. A. Golovchenko, Science 232, 48 (1986).

${ }^{5}$ R. J. Hamers, R. M. Tromp, and J. E. Demuth, Phys. Rev. Lett. 56, 1972 (1986).

${ }^{6}$ Quo-Xin Qian and D. J. Chadi, Bull. Am. Phys. Soc. 31, 584 (1986); J. E. Northrup, Bull. Am. Phys. Soc. 31, 584 (1986).

${ }^{7}$ J. R. Patel, J. A. Golovchenko, J. C. Bean, and R. C. Morris, Phys. Rev. B 31, 6884 (1985).

${ }^{8}$ P. L. Cowan, J. A. Golovchenko, and M. F. Robins (unpublished).

9 J. A. Golovchenko, J. R. Patel, D. R. Kaplan, P. L. Cowan, and M. J. Bedzyk, Phys. Rev. Lett. 49, 560 (1982).

${ }^{10}$ B. W. Batterman, Phys. Rev. 133, A759 (1964), and Phys. Rev. Lett. 22, 703 (1969).

${ }^{11}$ J. A. Golovchenko, W. L. Brown, and B. W. Batterman, Phys. Rev. B10, 4239 (1974).

${ }^{12}$ S. K. Andersen, J. A. Golovchenko, and G. Mair, Phys. Rev. Lett. 37, 1141 (1976).

${ }^{13}$ A. Ourmazd, D. Taylor J. Bevk, B. A. Davidson, L. C. Feldman, and J. P. Mannaerts, Phys. Rev. Lett. 57, 1332 (1986).

${ }^{14}$ R. S. Becker, J. A. Golovchenko, G. S. Higashi, and B. S. Swartzentruber, Phys. Rev. Lett. 57, 1020 (1986).

${ }^{15}$ H. J. Gossmann, J. C. Bean, L. C. Feldman, E. G. McRae, and I. K. Robinson, Phys. Rev. Lett. 55, 1106 (1985).

${ }^{16}$ H. J. Gossman and L. C. Feldman, Surf. Sci. 155, 413 (1985).

${ }^{17}$ S. M. Durbin, L. E. Berman, B. W. Batterman, and J. M. Blakely, Phys. Rev. B 33, 4402 (1986).

${ }^{18}$ R. I. G. Uhrberg, R. D. Bringans, M. A. Olmstead, R. Z. Bachrach, and J. E. Northrup, Bull. Am. Phys. Soc. 31, 373 (1986).

${ }^{19}$ A. Ishizaka, N. Nakagawa, and Y. Shiraki, in Proceedings of the Second International Symposium on Molecular Beam Epitaxy and Related Clean Surface Techniques (Japan Society of Applied Physics, Tokyo, 1982), p. 183.

${ }^{20}$ S. M. Durbin, L. E. Berman, B. W. Batterman, and J. M. Blakely, Phys. Rev. Lett. 56, 236 (1986).

${ }^{21}$ R. S. Becker, J. A. Golovchenko, and B. S. Swartzentruber, Phys. Rev. B 32, 8455 (1985).

${ }^{22}$ K. Takayanagi, Bull. Am. Phys. Soc. 31, 568 (1986). 